\title{
CT diagnosis of splenic vein occlusion: imaging features, etiology, and clinical manifestations
}

\author{
C. S. Marn, K. A. Edgar, I. R. Francis \\ Department of Radiology, University of Michigan Hospitals, 1500 E. Medical Center Drive, Room B1D520, Ann Arbor, MI \\ 48109-0030, USA
}

Received: 30 August 1993/Accepted: 12 October 1993

\begin{abstract}
Background: Previous reports have described the computed tomographic (CT) appearance of collateral veins following splenic vein occlusion (SVO). This retrospective study was performed to determine the etiology, clinical manifestations, and accuracy of CT diagnosis in patients with this entity.

Methods: A computer search of radiology reports for a 1-year period found 52 patients with SVO diagnosed by absence of visualization of the splenic vein accompanied by the formation of the expected perigastric collateral veins. Clinical data were reviewed for sequela of SVO and clinical impact of the diagnosis.

Results: In 12 cases, other studies confirmed the CT diagnosis of SVO. In no case was the CT diagnosis proved to be incorrect by other imaging studies. Angiographic records found five additional cases with SVO not diagnosed by CT, but two of five had convincing CT evidence of SVO noted upon reevaluation by the authors. Review of clinical data showed heme-positive stool in six, of which three had significant gastrointestinal hemorrhage. Splenic infarction occurred in two cases.

Conclusions: Our data indicate that SVO is more common than previously suspected and usually remains clinically silent, but CT appears to be highly specific and fairly sensitive for its diagnosis.
\end{abstract}

Key words: Pancreas, angiography - Abdomen, CT Pancreas, neoplasms - Splenic vein occlusion, diagnosis.

Contrast-enhanced abdominal computed tomography (CT) is frequently used for a wide variety of clinical

Correspondence to: C. S. Marn problems, and the use of relatively large amounts of iodinated contrast material administered in a bolus fashion allows for a noninvasive evaluation of vascular structures, including the portal venous system. Unfortunately, the evaluation of the splenic vein is sometimes difficult since this structure runs nearly parallel to the axial imaging plane. Nonvisualization of the splenic vein can be due to compression or thrombosis, or may simply be due to slice misregistration or poor timing of the contrast bolus. For many years, angiographers have used a well-defined pattern of perigastric collateral veins to support the diagnosis of splenic vein occlusion (SVO) when the splenic vein was not visualized during studies of the portal venous system [1,2]. SVO is often accompanied by increased flow and formation of varices in short gastric veins between the stomach and spleen, an enlarged gastroepiploic vein along the greater curve, and varices in the gastrohepatic ligament. In a previous study, we have shown that these collateral veins are often evident by CT in patients with SVO [3]. Thus, a CT study with nonvisualization of the splenic vein accompanied by perigastric collateral veins likely indicates the presence of SVO.

A traditional surgical view of SVO before 1970 indicated that this is a rare disorder which often manifests in gastrointestinal bleeding [4]. Increasing use of viseral angiography has tempered that view somewhat, indicating that the disorder may have been previously underdiagnosed [5]. If CT can accurately make this diagnosis, it would provide a noninvasive method to screen for SVO in a large patient population scanned for a wide variety of clinical indications. In this way, the true incidence and clinical significance of this disorder could be assessed.

The goals of this study are twofold: First, we attempted to measure the accuracy of the CT diagnosis of SVO based on nonvisualization of the splenic vein and 

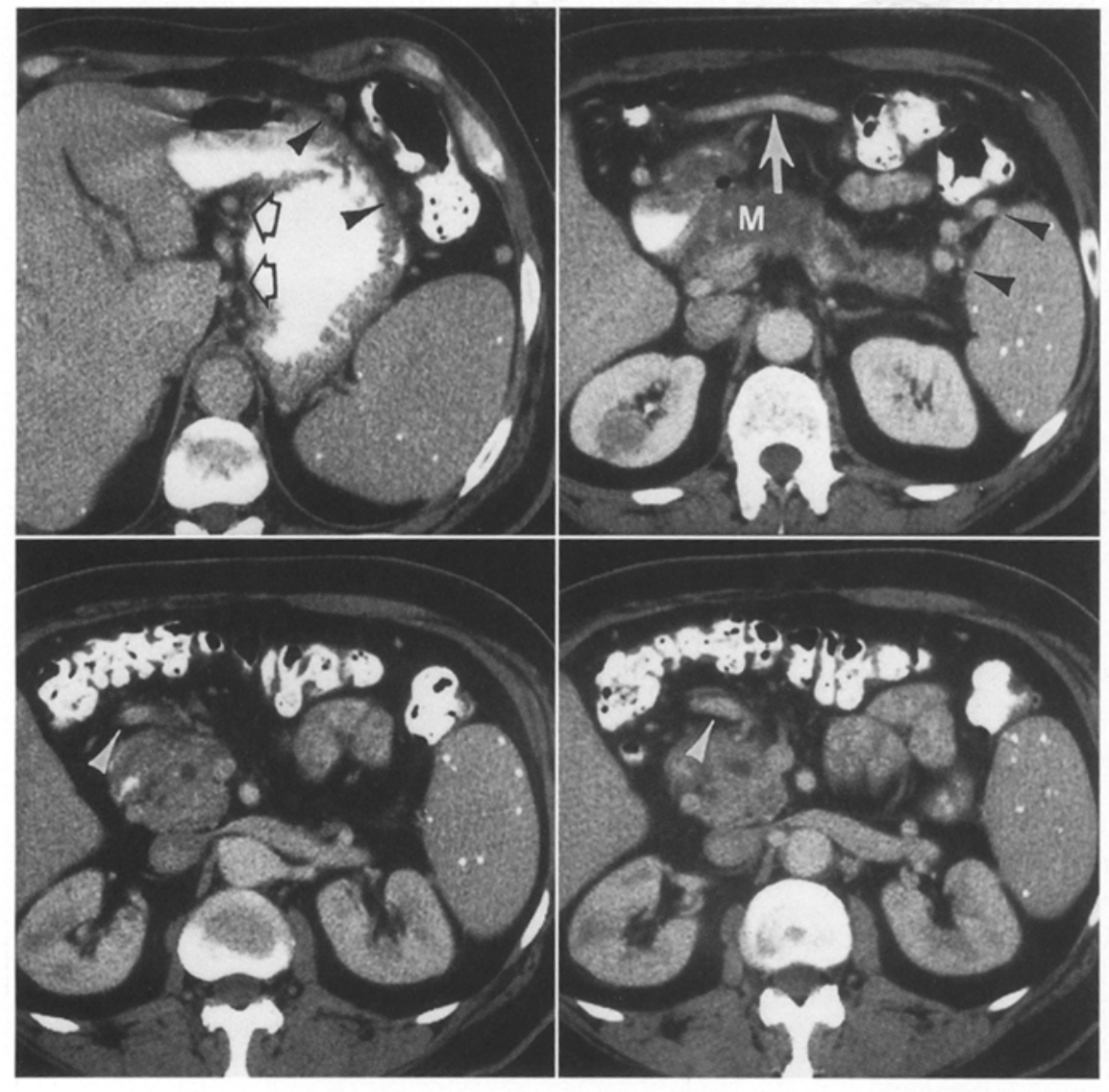

Fig. 1. CT of SVO. Four noncontiguous contrast-enhanced CT images in a patient with a pancreatic mass $(M)$ show a segment of splenic vein which does not enhance posterior to mass. Typical collaterals for SVO include varcies in the gastrohepatic ligament (open arrows), short gastric varices (black arrowheads), and an enlarged gastroepiploic vein (white arrow) which terminates in gastrocolic trunk (white arrowheads) anterior to head of pancreas. detection of perigastric collateral veins. Second, we wished to measure the frequency and impact of this diagnosis in a large patient population seen in a tertiary care center.

\section{Materials and Methods}

A computer search of radiology reports over a 1-year period was performed to identify cases of SVO diagnosed by CT, magnetic resonance imaging (MRI), or angiography. For those patients in whom the diagnosis was made by $\mathrm{CT}$, the scans were reviewed for evidence of nonvisualization of the splenic vein and the presence of collateral venous structures. Collateral veins were scored at three sites: large gastric veins between the stomach and spleen, left and right gastric veins draining in the gastrohepatic ligament, and enlarged gastroepiploic vein draining along the greater curve of the stomach. Criteria for enlargement of these vessels is based on previous research. When surpigenous, tubular structures in the gastrohepatic ligament were identified measuring greater than $6 \mathrm{~mm}$ in size, the study was considered positive for varices in the gastrohepatic ligament [6]. Similarly, short gastric varices were considered present when tubular structures greater than $5 \mathrm{~mm}$ were seen between the stomach and spleen, away from the splenic hilum [3]. The gastroepiploic vein was considered abnormal if it could be mapped from the splenic hilum to the gastrocolic trunk, and measured greater than $6 \mathrm{~mm}[3,7]$. In this patient population, evidence for collaborating studies was also sought (MRI, angiography, or surgery). Additionally, evidence of false negative CT studies was sought by reviewing cases where an angiographic diag- nosis of SVO was made, but that diagnosis was not made on a CT scan obtained near the time of angiography. The clinical records for the patient population were also reviewed for the clinical diagnosis, the presence of documented gastrointestinal bleeding, and the clinical impact of SVO.

All CT scanning was performed on a GE 9800 HiLight Advantage scanner (GE Medical Systems, Milwaukee, WI, USA). Studies were routinely performed utilizing $10-\mathrm{mm}$-thick contiguous axial sections. In those cases with a high suspicion of pancreatic disease identified before the CT scan, studies were performed utilizing 5-mm-thick contiguous sections through the region of the pancreas. Bolus infusion of $100-150 \mathrm{ml}$ of $60 \%$ meglumine iothalamate was performed at a rate of $2 \mathrm{~mL} / \mathrm{s}$, with scanning initiated $30-45 \mathrm{~s}$ after the start of the bolus. Oral contrast material was routinely administered in all cases.

\section{Results}

Fifty-two patients had the CT diagnosis of splenic vein occlusion. These were prospective diagnoses made by 10 different radiologists at our institution, based on nonvisualization of the splenic vein, and the development of the expected collateral veins as demonstrated in our previous report (Fig. 1) [3]. Review of the CT scans for presence of collateral vessels identified eight patients with other disorders which made the reliable identification of collaterals difficult. This included four patients with portal hypertension, three patients with upper ab- 
dominal adenopathy, and one patient with massive anasarca. The remaining 44 patients formed the basis of the patient group prospectively identified with SVO by $\mathrm{CT}$. The causes of SVO included pancreatic adenocarcinoma $(\mathrm{N}=23)$, pancreatitis $(\mathrm{N}=13)$, lymphoma $(\mathrm{N}$ $=2$ ), retroperitoneal abscess $(\mathrm{N}=2)$, and one case each of retroperitoneal sarcoma, pancreatic trauma, hypercoagulable state, and pancreatic mass without histological diagnosis. Table 1 gives the distribution of varices. Forty-one patients had varices in at least two of the three sites, with 24 patients having varices at all three sites. In 12 cases, an appropriate collaborating study was identified. This included angiography $(\mathrm{N}=8), \mathrm{MRI}(\mathrm{N}$ $=3$ ), and surgery $(\mathrm{N}=1)$. In most cases, the confirmatory study was performed within 2 weeks (range $=0$ 48 days, mean $=17$ days). In all cases, the additional study confirmed the CT diagnosis of SVO.

The review of the clinical records of the 44 patients with isolated splenic vein occlusion revealed hemepositive stool in six patients, three with significant gastrointestinal bleeding requiring transfusion. Gastrointestinal hemorrhage occurred 11 weeks before the CT diagnosis in one case, 5 weeks after the CT diagnosis in one case, and at the time of CT diagnosis in one case. In two cases, CT scans demonstrated a wedge-shaped splenic infarction, which was clinically silent. In all cases of pancreatic cancer, the tumor was found to be unresectable based on CT criteria. However, in every case multiple abnormalities were present to indicate unresectability (adenopathy, hepatic metastatic disease, and arterial invasion), and in no case was SVO the sole cause of unresectability.

The search of angiography records identified five cases in which SVO was demonstrated by angiography, but a CT scan performed near the time of angiography was interpreted without mention of SVO. Retrospective review of these cases by two of the authors (CSM, KAE) showed convincing CT evidence of SVO in two, leaving three cases as false negative examinations.

\section{Discussion}

Prior to 1970 , a traditional surgical view of isolated SVO held that this was a rare disorder, usually manifesting in gastrointestinal hemorrhage. Sutton et al. identified 53 cases of SVO in the English literature from 1900-1968 [4]. In these cases, upper gastrointestinal bleeding occurred in approximately $64 \%$. That series stressed the importance of splenoportography in the diagnosis of SVO. By 1985, it was recognized that celiac angiography could replace splenoportography as the definitive diagnostic tool for SVO. Moosna and Gadd [5] emphasized that SVO should be suspected in patients with pancreatitis and gastrointestinal blood loss, patients with splenomegaly in the absence of portal hyperten-
Table 1. Distribution of varices in 44 patients with SVO

\begin{tabular}{cccc}
\hline & \multicolumn{2}{l}{ Location of varices on CT } \\
\cline { 2 - 4 } No. of cases & GEV & SG & GHL \\
\hline 24 & + & + & + \\
9 & + & + & + \\
7 & & + & + \\
2 & + & & + \\
1 & 34 & + & \\
1 & & 41 & 34 \\
\hline
\end{tabular}

sion, cirrhosis, or hematologic disease, and patients with isolated gastric varices. In 1982, Keith et al. reported another series of patients with gastric varices secondary to SVO from pancreatic disease. These authors stressed the importance of identifying the triad of dilatation of the gastroepiploic vein, enlargement of the spleen with a normal liver, and associated gastric or gastroesophageal varices as a highly suggestive pattern for SVO. Numerous authors have emphasized that SVO must be differentiated from portal hypertension, since the surgical treatment is different $[5,8]$. Bleeding from varices secondary to SVO can be managed with splenectomy, while bleeding from portal hypertension with varices must be managed with the creation of a portosystemic shunt. The increased use of angiography has led to a significant increase in the diagnosis of SVO $[5,8]$.

More recently, Bernades et al. reported that in 266 patients with chronic pancreatitis, 35 were found to have splenic and/or portal vein occlusion by ultrasound, with subsequent confirmation by angiography and computed tomography [9]. Only one patient in this series was operated on for massive esophageal variceal bleeding, and another patient died due to colonic variceal bleeding. These authors concluded that the prevalence of the splenoportal venous obstruction in this large series of patients with pancreatitis was $13 \%$, but that bleeding is an unusual complication.

In 1990, our group reported that contrast-enhanced CT scans can frequently give both direct and indirect evidence of SVO. A pattern approach was developed, including the nonvisualization of the splenic vein, along with the identification of collateral venous pathways expected for this disorder. The purpose of our current study was to apply these imaging parameters to a large, unselected patient population undergoing CT for a variety of processes.

The results of our series support the concept that SVO is much more common than previously thought, and that gastrointestinal hemorrhage is an unusual manifestation of this disorder. In our large tertiary teaching hospital with patients referred for complex pancreatic disease, 44 patients were identified utilizing our previ- 
ously described CT criteria in a 1-year period. In keeping with previous reports, pancreatic cancer and pancreatitis were the major causes of this SVO. Of 44 patients, only three had significant gastrointestinal hemorrhage, and two patients had splenic infarction. It is now clear that the traditional view of SVO as a rare disorder is erroneous. More correctly, SVO is a common feature of many pancreatic, retroperitoneal, or hematologic processes, but it is usually clinically silent.

Given the selection criteria utilized for this study, it is difficult to calculate values for sensitivity and specificity of CT for the diagnosis of SVO. However, some conclusions can be drawn. Over this 1-year period, only five cases from angiography records could be identified where an SVO was documented by angiography, but not reported on CT. Retrospective review of these cases indicate that there was adequate evidence to support the diagnosis of SVO by CT on two studies, leaving three CT studies as false negative examinations. CT does appear to be highly specific for the diagnosis, given the evidence collected in this series. While a confirmatory examination was available in only 12 of 44 cases, in no case was a CT interpretation of SVO found to be incorrect.

In conclusion, SVO should be suspected when the splenic vein is not visualized, and the expected perigas- tric collateral veins are present. SVO is frequently clinically silent, and variceal hemorrhage is an uncommon manifestation of SVO.

\section{References}

1. Salam AA, Warren WD, Tyras DH. Splenic vein thrombosis: a diagnosable and curable form of portal hypertension. Surgery 1973;74:961-972

2. Walter JF, Chuang VP, Bookstein JJ, Reuter SR, Cho KJ, Pulmano $\mathrm{CM}$. Angiography of massive hemorrhage secondary to pancreatic disease. Radiology 1977;124:337-342

3. Marn CS, Glazer GM, Williams DM, Francis IR. CT-angiographic correlation of collateral venous pathways in isolated splenic vein occlusion: new observations. Radiology 1990;175:375-380

4. Sutton JP, Yarborough CY, Richards JT. Isolated splenic vein occlusion. Arch Surg 1970;100;623-626

5. Moossa AR, Gadd MA. Isolated splenic vein thrombosis. World $J$ Surg 1985;9:384-390

6. Balfe DM, Mauro MA, Koehler RE, et al. Gastrohepatic ligament: normal and pathologic CT anatomy. Radiology 1984;150:485-490

7. Moody AR, Poon PY. Gastroepiploic veins: CT appearance in pancreatic disease. AJR 1992;158:779-783

8. Keith RG, Mustard RA, Saibil EA. Gastric variceal bleeding due to occlusion of splenic vein in pancreatic disease. Can J Surg $1982 ; 25: 301-304$

9. Bernades P, Baetz A, Levy P, Belghiti J, Menu Y, Fekete F. Splenic and portal venous obstruction in chronic pancreatitis: a prospective longitudinal study of a medical-surgical series of 266 patients. Dig Dis Sci 1992;37:340-346 